Ann. Sci. forest., 1978, 35 (3), 203-212.

\title{
Péricarpe et cinétique de germination des glands de chêne pédonculé
}

\author{
M. LAMOND \\ Laboratoire de Phyfomorphogenèse $n^{\circ} 45$, Ass. au CNRS, \\ 4, rue Ledru, 63000 Clermont-Ferrand, France
}

\begin{abstract}
Résumé
Analyse de la cinétique de germination des glands de chêne pédonculé (Quercus pedunculata Ehr.) conservés en tourbe sèche, à basse température.

L'ablation du péricarpe et des téguments ainsi qu'un lavage à l'eau courante des embryons dénudés stimulent fortement la vitesse de germination de cette semence. L'imbibition des glands entiers, avant leur mise en germoir, n'a par contre aucun effet sur leur germination.

L'ablation des enveloppes séminales, suivie d'un lavage de $48 \mathrm{~h}$ des embryons dénudés, permet une synchronisation maximum de la germination. Ce traitement est sans aucune influence sur la croissance et le développement ultérieurs des jeunes plantes de cette espèce.
\end{abstract}

\section{Introduction}

De nombreux travaux orientés vers la sylviculture (Bonnet-Masimbert et Muller, 1973 ; Cornu ef al., 1973 ; Croizeau et Roguet, 1976 ; Garbaye et Bonneau, 1975 ; Garbaye et Leroy, 1974 ; ...) ou la physiologie du développement (Bonner, 1968 ; Farmer Jr, 1975 ; Men, 1976 ; Pellecuer, 1976 ; Suszka et Krawiarz, 1971 ; ...) ont été entrepris au cours des dernières années en vue de remédier à la régénération insuffisante des peuplements de chêne. Ce problème résulte en grande partie de l'hétérogénéité des glandées, de l'impossibilité de conserver convenablement les glands sur de longues périodes ainsi que du manque de compétitivité et de la faible croissance des jeunes plantes issues de semis.

Au cours de nos recherches sur la morphogenèse du système racinaire du chêne pédonculé, nous nous sommes heurté à de très sérieuses difficultés pour obtenir des semis d'âge physiologique comparable. La germination des semences de cette espèce, en particulier lorsqu'il s'agit de glands bien ressuyés et conservés au sec à basse température, s'étale le plus souvent sur une période de 30 à 50 j, ce qui entraîne chronologiquement une très forte hétérogénéité dans le stade de développement des semis.

Pour résoudre ce problème et en nous inspirant des techniques de « test 》 rapide de germination décrites par Sanesi (1968), nous avons étudié l'effet de quelques traitements physiques simples, antérieurs à la mise en germoir, sur la cinétique de germination de ces glands et la croissance ultérieure des plantes qu'ils engendrent. 


\section{Matériel et méthodes}

Les essais ont tous été effectués sur des glands de chêne pédonculé (Quercus pedunculata Ehrl.) récoltés en octobre 1976, soit sous un même arbre isolé en Creuse, soit sous divers arbres isolés dans la région de Lezoux (Puy-de-Dôme). Ces glands avaient une teneur en eau à la récolte d'environ 40 p. 100 par rapport à leur poids frais. Ils ont été stratifiés jusqu'à utilisation ( 3 à 5 mois), à environ $3^{\circ} \mathrm{C}$, dans de la tourbe sèche.

Au sein d'une même expérience, nous avons toujours utilisé des glands provenant d'un même arbre, récoltés à la même date et conservés dans le même bac. Dans tous les cas, ces derniers ont été mis à germer verticalement (hile vers le haut), à demi enfouis dans de la vermiculite saturée d'eau, à l'intérieur de bacs de plastique recouverts de papier d'aluminium et placés en chambre obscure à température rigoureusement contrôlée.

Dans un premier groupe d'expériences nous avons comparé la cinétique de germination de lots de cinquante glands ayant subi, avant leur mise en germoir, l'un ou l'autre des traitements suivants:

- aucun traitement (glands intacts) ;

micropile à leur sortie de tourbe ;

(T-48) - immersion de $48 \mathrm{~h}$ dans l'eau courante ;

(T-48-AP) - immersion de $48 \mathrm{~h}$ dans l'eau courante suivie de l'ablation des enveloppes séminales (péricarpe et téguments) ;

(AP) - ablation des enveloppes séminales et mise en germoir sans immersion dans l'eau courante ;

(AP-48) - ablation des enveloppes séminales suivie d'un lavage de $48 \mathrm{~h}$ à l'eau courante des embryons dénudés.

Ces essais ont tous été poursuivis durant 24 j avec relevé du nombre de semences germées dans chaque lot tous les $4 \mathrm{j}$. Nous avons utilisé comme critère de germination les premiers signes visibles de croissance du pivot : émergence de ce dernier à travers le micropile dans le cas des glands intacts, allongement d'environ $5 \mathrm{~mm}$ dans tous les autres cas.

La sortie des glands de leur bac de conservation à basses températures fut considérée comme temps origine pour tous les lots de semences, indépendamment du traitement subi par ces dernières avant leur mise en germoir au sens strict.

Dans le but de préciser la durée optimale du lavage des semences dénudées pour obtenir une levée de croissance homogène des jeunes plants, nous avons cultivé à $24^{\circ} \mathrm{C}$, sur vermiculite saturée d'eau, des lots de cinquante embryons préalablement immergés dans l'eau courante durant $0,24,48$ et $96 \mathrm{~h}$. Douze jours après la mise en culture, nous avons mesuré la longueur des pivots dans chacun des lots et comparé les variances intra-lots de ce paramètre.

Afin de vérifier l'influence possible, des divers traitements appliqués aux glands avant la mise en germoir, sur la croissance et le développement ultérieur des semis, nous avons cultivé sur caisson à aérosol nutritif (Lamond, 1975), à $25^{\circ} \mathrm{C}$ et sous un éclairement quotidien de $16 \mathrm{~h}\left(24000 \mathrm{ergs} . \mathrm{cm}^{2}\right)$, les dix premières germinations obtenues dans chacun des lots de l'une de nos expériences précédentes. Ces plantules furent repiqués sur le caisson lorsque leur pivot mesurait entre 8 et $10 \mathrm{~cm}$ de longueur. 
Au cours de cette expérience, le poids frais total de chacun des semis de même que la longueur des épicotyles et des pivots furent mesurés toutes les semaines. Après $28 \mathrm{j}$ de culture, tous les plants ayant complété la croissance et le développement de leur premier étage de feuilles, nous avons évalué en outre les paramètres suivants : nombre de feuilles; diamètre au collet; poids frais des tiges feuillées, des cotylédons et des systèmes racinaires.

\section{Résultats obtenus}

a) Cinétiques de germination

Les résultats de nos expériences sur la germination des glands de chêne pédonculé sont résumés au tableau 1 (TA. 1) sous forme de T-50 (temps nécessaire à l'obtention de 50 p. 100 de germination). Ce mode d'expression de la vitesse de germination illustre assez bien les différences de cinétique que nous avons observées à la suite des divers traitements infligés à ces semences avant leur mise en germoir.

\section{TABLEAU 1}

TA. 1. - Temps (jours) nécessaire à l'obtention de 50 p. 100 de germination en fonction du traitement appliqué aux glands avant leur mise en germoir: (T), aucun traitement; (T-PA), glands dont le pivot émergeait à travers le micropile ; (T-48), immersion de $48 \mathrm{~h}$; (T-48-AP), immersion de $48 \mathrm{~h}$ suivie de l'ablation des enveloppes séminales ; (AP), ablation des enveloppes séminales ; (AP-48), ablation des enveloppes séminales suivie d'un lavage de $48 \mathrm{~h}$ des embryons nus

TA. 1. - Time (days) required to allow 50 p. 100 of the seeds to germinate after the different treatments performed to the acorns before their incubation: (T), any treatment ; (T-PA), acorns with emerging radicle; (T-48), $48 \mathrm{hrs}$ soaking of the acorns in running water; (T-48-AP), 48 hrs soaking followed by the removal of the testae; (AP), removal of the testae; (AP-48), removal of the testae followed by a $48 \mathrm{hrs}$ soaking of the embryos in running water

\begin{tabular}{|c|c|c|c|c|c|c|c|c|}
\hline \multicolumn{2}{|c|}{ Récolte des glands } & \multirow{2}{*}{$\begin{array}{c}\text { Température } \\
\text { de } \\
\text { germination }\end{array}$} & \multicolumn{6}{|c|}{ Traitement effectué } \\
\hline Provenance & Date & & $T$ & T-PA & $\mathrm{T}-48$ & $\mathrm{~T}-48-\mathrm{AP}$ & AP & AP- 48 \\
\hline Creuse & $17 . \times .76$ & $16^{\circ} \mathrm{C}$ & 19,8 & - & 15,8 & - & 3,6 & 2,0 \\
\hline- & - & $24^{\circ} \mathrm{C}$ & 22,0 & - & 14,0 & - & 2,7 & 2,0 \\
\hline - & - & $24^{\circ} \mathrm{C}$ & 15,4 & 6,8 & 21,5 & 5 & 2,3 & 2,0 \\
\hline Puy-de-Dôme & $16 . \times .76$ & $20 \circ \mathrm{C}$ & $24<$ & - & $24<$ & - & 2,6 & 2,0 \\
\hline- & $23 . \times .76$ & $24 \circ \mathrm{C}$ & 20,0 & - & 20,5 & - & 3,2 & 2,2 \\
\hline
\end{tabular}

L'on notera principalement que, dans toutes ces expériences, les semences mises à germer après ablation des enveloppes séminales (Lots: T-48-AP, AP et AP-48) germent beaucoup plus rapidement que les autres (Lots : T, T-48 et T-PA). Ce traitement est un peu moins efficace lorsqu'il est effectué après imbibition des glands (T.48-AP) ; un lavage de $48 \mathrm{~h}$ à l'eau courante des embryons dénudés (AP-48) stimule par contre davantage la germination que la seule ablation du péricarpe et des téguments (AP).

L'immersion des glands entiers dans l'eau pendant $48 \mathrm{~h}$ semble n'avoir aucune influence généralisable sur leur germination. Dans deux expériences ce traitement eut pour effet de raccourcir notablement le T-50 mais ce résultat ne s'est pas confirmé 
par la suite. De plus, si l'on utilise « l'inverse X 100 » (Harrington, 1962) du « coefficient de vélocité » de Kotowski (1926) comme indice de la vitesse de germination, dans tous ces essais les valeurs obtenues sur les lots de glands immergés $48 \mathrm{~h}$ dans l'eau sont légèrement supérieures à celles des témoins.

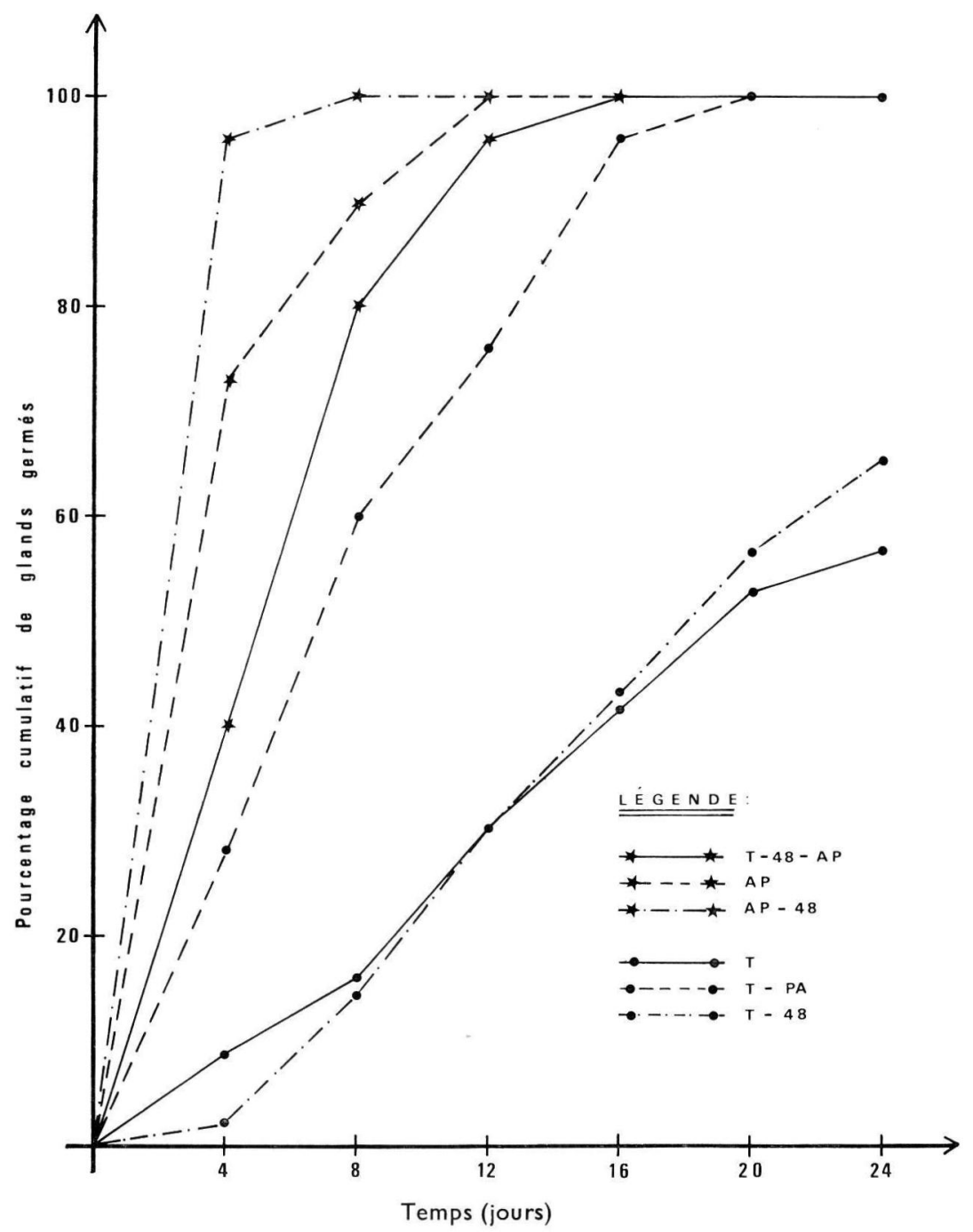

FIG. 1. - Courbes de germination en fonction du temps de l'ensemble des glands mis à germer à $24^{\circ} \mathrm{C}$ à la suite de l'un ou l'autre des traitements suivants : (T), aucun traitement; (T-PA), aucun traitement mais glands dont le pivot émergeait à travers le micropile; (T-48), immersion de $48 \mathrm{~h}$ dans l'eau courante ; (T-48-AP), immersion de 48 h dans l'eau courante suivie de l'ablation des enveloppes séminales ; (AP), ablation des enveloppes séminales et mise en germoir sans immersion préalable dans l'eau ; (AP-48), ablation des enveloppes séminales suivie d'un lavage de 48 h des embryons dénudés.

Cumulative germination curves of acorns incubated at $24^{\circ} \mathrm{C}$ after one or the other of the following treatments : (T), any treatment; (T-PA), acorns with emerging radicle ; (T-48), 48 hrs soaking of the acorns in running water; (T-48-AP), 48 hrs soaking followed by the removal of the testae ; (AP), removal of the testae ; (AP-48), removal of the testae followed by a 48 hrs soaking of the embryos. 
Les courbes de germination en fonction du temps (fig. 1) de l'ensemble des lots placés à $24^{\circ} \mathrm{C}$ illustrent tant cet effet négligeable de l'immersion des glands entiers (chevauchement des courbes $T$ et T-48), que l'influence considérable de l'ablation des enveloppes séminales (pente très forte des courbes AP-48, AP et T-48-AP) sur la cinétique de germination des semences de chêne pédonculé.

\section{b) Durée optimale de l'immersion des embryons dénudés}

Les moyennes arithmétiques et les variances de longueur de pivots des semis de $12 \mathrm{j}$, obtenus à la suite d'immersions de durée variable dans l'eau courante de lots de cinquante embryons, sont groupés au tableau 2 (TA. 2).

\section{TABLEAU 2}

TA. 2. - Longueur moyenne $(\bar{x})$ et variance des longueurs $\left(S^{2} x\right)$ de pivots, $12 j$ après la mise en germoir à $24^{\circ} \mathrm{C}$, en fonction de la durée de l'immersion préalable des embryons dans l'eau courante : I'astérisque indique une variation significative au seuil de 5 p. 100 de variances considérées

TA. 2. - Mean length $(\bar{x})$ and lengths' variance $\left(S^{2} x\right)$ of radicles after 12 days of incubation a $24^{\circ} \mathrm{C}$ of embryos previously soaked in running water during different period of time. Asterisks indicate a significant difference of the variances at the 5 p. 100 level

\begin{tabular}{|c|c|c|c|c|}
\hline \multirow{3}{*}{$\begin{array}{c}\text { Durée de } \\
\text { l'immersion } \\
\text { (Hrs) }\end{array}$} & \multicolumn{4}{|c|}{ Récolte de glands } \\
\hline & \multicolumn{2}{|c|}{$L$ et $L-17 . X .76$} & \multicolumn{2}{|c|}{$\mathrm{L}$ et $\mathrm{L}-23 . \times .76$} \\
\hline & $\bar{x}$ & $\mathrm{~S}^{2} \mathrm{x}$ & $\bar{x}$ & $S^{2} x$ \\
\hline $\begin{array}{r}0 \\
24 \\
48 \\
96\end{array}$ & $\begin{array}{l}9,082 \\
8,687 \\
8,438 \\
7,842\end{array}$ & $\begin{array}{c}10,727 * \\
7,402 \\
6,629 * \\
7,384\end{array}$ & $\begin{array}{c}7,696 \\
\overline{9,792} \\
-\end{array}$ & $\begin{array}{c}19,954 * \\
\overline{12,087} * \\
-\end{array}$ \\
\hline
\end{tabular}

L'on remarquera que pour les deux récoltes étudiées ( $L$ et $L-17 . X .76$ et $L$ ef $L-23 . X .76)$, la variance des longueurs de pivot des semis obtenus après une immersion de $48 \mathrm{~h}$ des embryons dans l'eau courante s'avère significativement plus faible, au seuil de 5 p. 100 , que celle des germinations n'ayant subi aucun lavage préalable.

Ceci montre bien que le lavage des embryons, avant leur mise en germoir, augmente l'homogénéité physiologique des semis. En outre, bien que l'on n'observe aucune différence significative entre les variances de longueur de pivot des lots de semis issus d'embryons lavés, on notera que cette variance atieint une valeur minimale pour une durée de $48 \mathrm{~h}$. L'élévation de cette valeur sur les semis obtenus après $96 \mathrm{~h}$ de lavage des embryons ainsi que l'augmentation du nombre de modes sur l'histogramme de fréquence de longueur des pivots de ce lot de semences (fig. 2) suggèrent qu'un lavage trop prolongé peut réduire à nouveau l'homogénéité physiologique des semis.

c) Croissance et développement des semis

Les divers traitements appliqués aux semences, avant leur mise en germoir, n'ont apparemment aucune influence sur la croissance et le développement ultérieur 

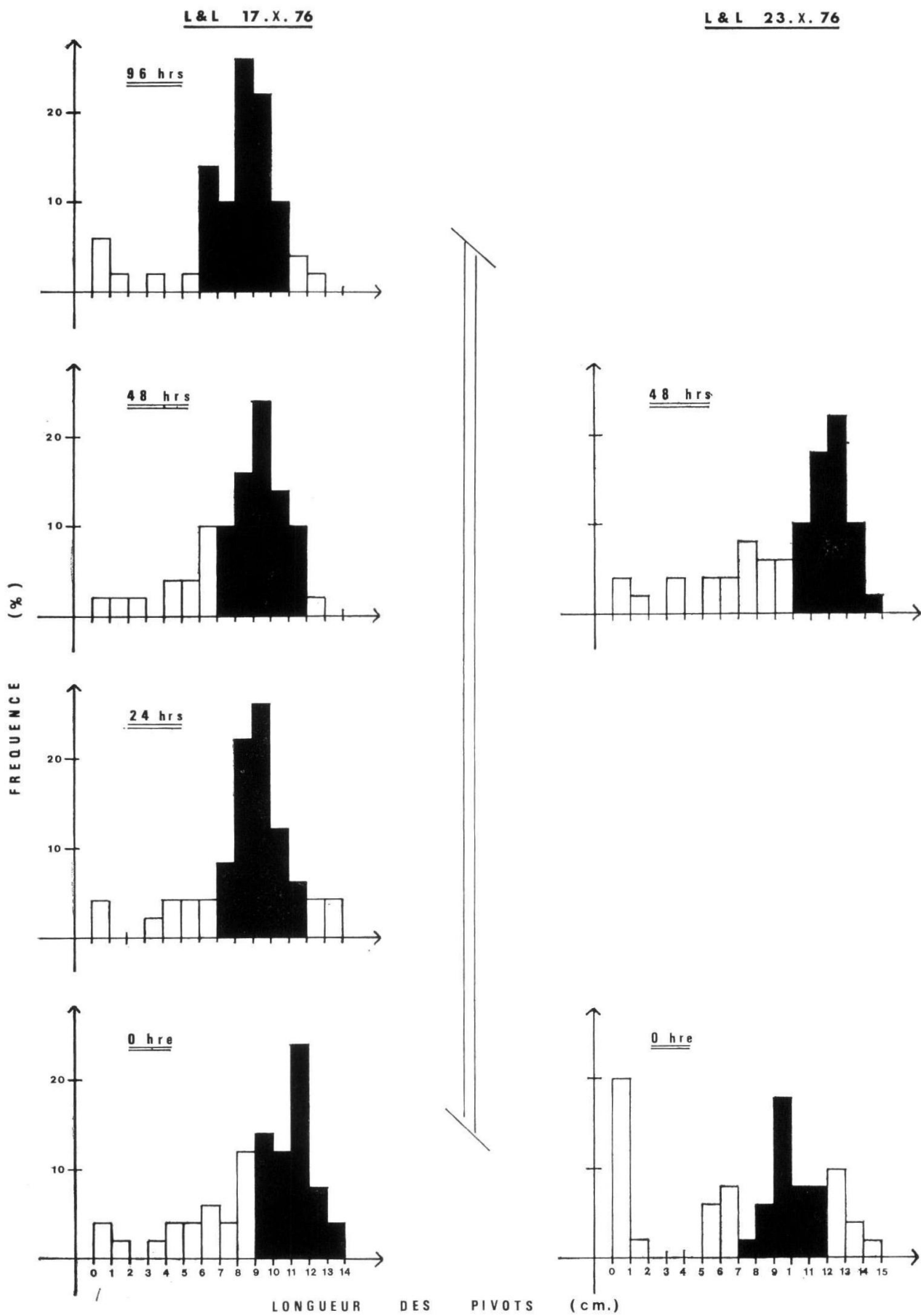
des semis, du moins jusqu'à la fin de la première vague de croissance des parties aériennes.

En effet, nous n'avons noté aucune différence dans la vitesse de croissance en longueur des épicotyles et des pivots ainsi que dans l'augmentation hebdomadaire de poids des plants cultivés à partir des germinations de glands témoins ou de semences ayant subi l'un ou l'autre des pré-traitements étudiés.

L'analyse de variance, effectuée sur chacun des paramètres mesurés après $28 \mathrm{j}$ de culture à $25^{\circ} \mathrm{C}$ (nombre de feuilles; diamètre au collet; longueur des tiges et des pivots ; poids frais des plants entiers, des tiges, des racines et des cotylédons), ne montre aucune différence significative, au seuil de 5 p. 100, entre les échantillons étudiés.

Cette similitude quasi parfaite de croissance et de développement entre les plants de chacun des lots se trouve d'ailleurs très bien illustrée par le fort recouvrement des intervalles de confiance des moyennes arithmétiques des paramètres mesurés (fig. 3).

\section{Discussion et conclusions}

Les résultats obtenus au cours de ces expériences montrent clairement que le péricarpe et (ou) les téguments constituent un obstacle non négligeable à la germination rapide et homogène des glands de chêne pédonculé. Nous n'avons malheureusement pas encore réussi à mettre en évidence de façon précise le (ou les) mécanisme(s) à l'origine de cet effet dépressif des enveloppes séminales sur la germination de cette semence.

Des mesures préliminaires, effectuées à l'aide de l'appareil de Frossard (1975), indiquent que les glands entiers, immergés dans de l'eau agitée, consomment douze à vingt fois moins d'oxygène par gramme de matière sèche que des embryons dénudés placés dans les mêmes conditions. Le péricarpe et les téguments, du moins lorsqu'ils sont imbibés, constituent donc une barrière limitant considérablement la respiration de l'embryon. Le degré d'anoxie de l'atmosphère interne du gland semble en outre renforcé par la consommation oxydative d'oxygène au niveau des enveloppes séminales, comme dans le cas des graines de Pirus malus L. (Côme, 1967 ef̂ 1971), d'Hedysarum coronarium (Côme et Semadeni, 1973) et de Chenopodium bonus-henricus (Dorne et Côme, 1976).

FIG. 2. - Histogrammes de fréquence des longueurs de pivot obtenues, $12 \mathrm{j}$ après la mise en germoir, à partir d'embryons nus (récoltes $\mathrm{L}$ et $\mathrm{L}-17$. X.76 et $\mathrm{L}$ et $\mathrm{L}-23 . \mathrm{X} .76$ ) préalablement immergés 0,24 , 48, ou 96 h dans l'eau courante. Les cinq classes de longueur rattachées au mode principal de ces histogrammes ont été complètement noircies de façon à mieux faire ressortir le regroupement des longueurs de pivots à la suite de lavage des embryons. L'on notera la réapparition d'un second mode dans cette région à la suite d'un lavage de $96 \mathrm{~h}$.

Frequency histogram of radicles "length of 12 days old seedlings, incubated at $24^{\circ} \mathrm{C}$ after a removal of the acorns' testae followed by a $0,24,48$ or 96 hrs soaking of the embryos in running water. The principal groups and their rigth and left two adjoining one had been darken to bring out the regrouping of radicles' length in consequence of the washing of the embryos. Note also the reapparance of two modes in this area when the embryos are washed during 96 hrs. 

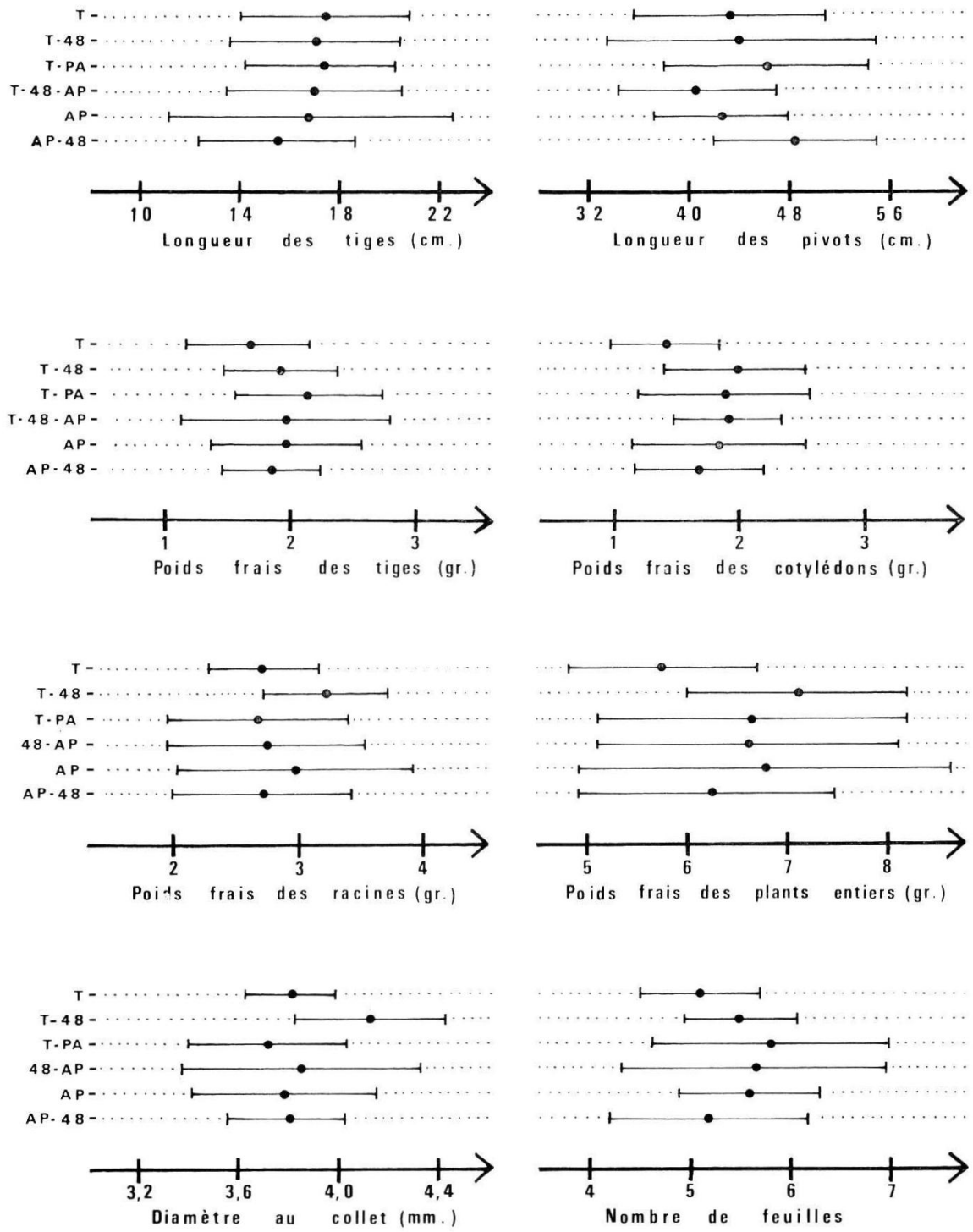

FIG. 3. - Moyennes ( $\bullet$ ) et intervalle de confiance des moyennes (-) des paramètres de croissance et de développement mesurés, après $28 j$ de culture (fin de la première vague de croissance des parties aériennes), sur des plants cultivés à partir de semis obtenus après l'un ou l'autre des traitements effectués sur les semences avant leur mise en germoir : (T), aucun traitement ; (T-PA), aucun traitement mais glands dont le pivot émergeait à travers le micropile ; (T-48), immersion des glands entiers dans l'eau courante pendant $48 \mathrm{~h}$; (T-48-AP), immersion de $48 \mathrm{~h}$ dans l'eau courante suivie de l'ablation des enveloppes séminales ; (AP), ablation des enveloppes séminales et mise en germoir sans immersion préalable dans l'eau ; (AP-48), ablation des enveloppes séminales suivie d'un lavage à l'eau courante de $48 \mathrm{~h}$ des embryons dénudés.

Confidence-internal (5 p. 100 level) of the means of the growth and development parameters measured, after 28 days of culture (end of the first flush growth of the aerial parts), on plants grown from germinated acorns who had undergone, before incubation, one or the other of the following treatments : $(T)$, any treatment ; (T-PA), acorns with emerging radicle; (T-48), 48 hrs soaking of the acorns in running water ; (T-48-AP), soaking followed by the removal of the testae; (AP), removal of the testae ; (AP-48), removal of the testae followed by a $48 \mathrm{hrs}$ soaking of the embryos in running water. 
La mauvaise germination des glands entiers pourraient en outre s'expliquer, du moins en partie, par une réhydratation déficiente des embryons. Watt (1919) a montré que l'extrémité micropilaire (seule en contact avec le substrat humide lorsque les glands sont mis à germer verticalement) jouait un rôle négligeable dans l'imbibition des glands de chêne pédonculé. Les travaux récents de Levert (1977) sur la réhydratation des semences de cette espèce confirment la très faible perméabilité hydrique de cette zone du péricarpe et montrent que l'embryon dénudé s'imbibe beaucoup mieux et beaucoup plus rapidement que le gland entier. La reprise d'eau par l'embryon lui-même, à l'intérieur de ses enveloppes séminales, ne débuterait en outre qu'après l'émergence de la radicule à travers le micropile.

L'effet stimulant d'une immersion de 48 h des embryons nus sur la germination ainsi que l'accroissement de l'homogénéité morpho-physiologique des semis à la suite de ce traitement renforcent cette hypothèse. La teinte jaunâtre de l'eau après quelques heures de trempage des embryons suggère cependant que ce lavage puisse avoir pour effet complémentaire d'éliminer, au moins en partie, un certain nombre de composés (peut-être des polyphénols oxydables comme chez le Pommier ou le Chénopode) susceptibles de nuire à la germination ou à la croissance ultérieure des semis.

L'absence de différence significative, à la fin de la première vague de croissance des parties aériennes, entre les plants issus de glands entiers et ceux qui proviennent d'embryons nus, lavés 48 h, montre bien que l'ablation des enveloppes, suivie d'un lavage des embryons, n'a aucune influence sur la croissance et le développement ultérieur des semis.

Ce traitement des semences, avant leur mise en germoir, ne fait que permettre à la majorité des embryons de germer en quelques jours et ainsi de débuter leur croissance sensiblement au même moment. II induit une très forte synchronisation de départ, qui élimine presque totalement l'hétérogénéité chronologique observée dans une population de jeunes plantes issues de glands entiers.

En résumé, l'ablation du péricarpe et des téguments, suivie d'un lavage de $48 \mathrm{~h}$ des embryons, constitue un moyen simple et efficace pour l'obtention rapide de semis homogènes et vigoureux à partir de glands de chêne pédonculé conservés à sec, à basse température. La faible adhérence de l'embryon à ses enveloppes ainsi que la forme régulière des semences de cette espèce devraient permettre de mécaniser l'opération de décorticage et l'appliquer dans la pratique forestière courante.

Reçu pour publication en décembre 1977.

\section{Remerciements}

L'auteur tient à remercier bien sincèrement le Ministère des Affaires Etrangères de France ainsi que la D.G.R.S.T. pour leur aide financière.

\section{Summary}

Influence of the testae upon the germination speed of acorns of the British common oak (Quercus pedunculata Ehrl)

This paper is mainly concerned with the effect of the testae upon the kinetic of germination of acorns of Quercus pedunculata Ehrl. that have been stored at low temperature in dry peat. 
Evidences are provided that a removal of the testae and a 48 hours wash of the embryo in running water allows more than ninety per cent of the acorns to germinate within 4 days. On the other hand, a previous soaking of the acorns does not change their germination speed or rate.

Soaking of the whole acorn as well as the removal of the testae and/or the washing of the embryo do not influence the further growth and development of the seedlings. A statistical analysis, performed at the end of the first flush growth of the aerial parts, did not show any significant difference in the total weight, number of leaves, length and weight of the stem and of the root system between plants grown from regular seedlings and from those obtained after one or the other of these acorns treatments.

\section{Références bibliographiques}

BONNER F. T., 1968. Water uptake and germination of red oak acorns. Bot. Gaz., 129: 83-85.

BONNET-MASIMBERT M., MULLER C., 1973. La conservation des faines et des glands. Recherches et perspectives. Bull. Tech. Office National des For., 5 : 13-19.

COME D., 1967. L'inhibition de germination des graines de pommier (Pirus malus L.) non dormantes. Rôle possible des phénols tégumentaires. Ann. Sc. Naturelles, Bot., 8 : 371-478.

COME D., 1971. Dégazage des enveloppes séminales lors de leur imbibition. II. Cas des graines de Pommier. Physiol. vég., $9: 447-452$.

COME D., SEMADENI A., 1973. Dégazage des enveloppes séminales lors de leur imbibition. III. Application à l'étude de la duretée des graines d'Hedysarum coronarium L. Physiol. vég., 11 : 171-179.

CORNU D., GARBAYE J., LE TACON F., 1975. Résultats d'un essai préliminaire sur le bouturage du chêne et du hêtre. Rev. Forest. Franc., 27 : 139-140.

CROIZEAU D., ROGUET M., 1976. Faculté de reprise des glands prélevés en forêt après germination. Rev. Forest. Frenc., 28 : 275-279.

DORNE A., COME D., 1976. Germination des semences de Chenopodium bonus-henricus. Recherche du mécanisme de l'inaptitude à la germination des semences récoltées à une altitude élevée. Biol. Plant., 18 : 72-77.

FARMER R. E., Jr., 1975. Growth and assimilation rate of juvenile northern red oak : effects of light and temperature. Forest. Sc., $21: 273-381$.

FROSSARD J. S., 1975. Relations entre l'éclairement des feuilles et l'absorption d'oxygène par les racines de tournesols (Helianthus annuus L.). In C.R. des Séminaires du Groupe d'Etude des Racines ; éd. par J. Gagnaire-Michard et A. Riedacker ; Grenoble, 2 : 212-224.

GARBAYE J., BONNEAU M., 1975. Premiers résultats d'un essai de fertilisation sur plantation de chêne rouvre (Quercus sessiliflora). Ann. Sc. forest., 32 : 175-183.

GARBAYE J., LEROY PH., 1974. Influence de la fertilisation sur la production de glands en forêt de Bercé et forêt de Boulogne. Rev. Forest. Franc., 26 : 223-228.

HARRINGTON J. F., 1962. The effect of temperature on the germination of several kinds of vegetable seeds. XVIth inter. Horticult. Cong. (Bruxelles), 2 : 435-441.

KOTOWSKI F., 1926. Temperature relations to germination of vegetable seeds. Proc. Amer. Soc. Hort. Sc., 23 : 176-184.

LAMOND M., 1975. Dispositif de culture de plantes entières en caissons, sous brouillard nutritif, en usage à Clermont-Ferrand. In C. R. des Séminaires du Groupe d’Ełude des Racines ; éd. par J. Gagnaire-Michard et A. Riedacker ; Grenoble, 2 : 6-33.

LEVERT J., 1977. Etude de l'influence de quelques facteurs physiques sur la germination des glands de chêne pédonculé (Quercus pedunculaia Ehri., syn. : Q. robur L.), D.E.A., Université de Clermont II.

MEN S., 1976. Recherches sur la biologie, la morphologie et les moyens de lutte contre l'agent de la " pourriture noire » des glands : le Ciboria batschiana (Zopf) Buckwald.

PELLECUER B., 1976. L'écologie des chênes en forêt de Morimond et ses applications sylvicoles. Thèse, Dr.-Ingénieur, Nancy.

SANESI G., 1960. Indagine su due metode rapidi per la determinazione della germinabilita delle ghiande. Ital. For. Mont., 15 : 190-192.

SUSZKA G., KRAWIARZ K., 1971. Preparation of non stored red oak seed (Quercus borealis Mich.) for germination. Arboretum Kornickie, 16 : 131-152.

WATT A. S., 1919. On the causes of failure of natural regeneration in British oakwoods. J. Ecol., 7 : 173-203. 